\title{
Roles of flanking sequences in the binding between unimolecular parallel-stranded G-quadruplexes and ligands
}

\author{
GAI Wei ${ }^{1,2}$, YANG QianFan $^{1 *}$, XIANG JunFeng $^{1}$, SUN HongXia $^{1,2}$, SHANG Qian $^{1}$, LI Qian $^{1}$, \\ JIANG Wei ${ }^{1}$, GUAN AiJiao ${ }^{1,2}$, ZHANG Hong ${ }^{1}$, TANG YaLin ${ }^{1 *} \&$ XU GuangZhi ${ }^{1}$ \\ ${ }^{1}$ Beijing National Laboratory for Molecular Sciences, Center for Molecular Sciences, State Key Laboratory for Structural Chemistry for Unstable \\ and Stable Species, Institute of Chemistry, Chinese Academy of Sciences, Beijing100190, China; \\ ${ }^{2}$ Graduate University of Chinese Academy of Sciences, Beijing100049, China
}

Received March 26, 2012; accepted May 21, 2012

\begin{abstract}
G-quadruplexes attract more and more attention in recent years. Numerous small molecules which can induce or stabilize the formation of G-quadruplexes have been investigated on the purpose of anticancer drug development. As a motif existed in physiological condition, flanking sequences are an important part of G-quadruplexes but the study on the impact of flanking sequences on (G-quadruplex)-ligand binding is rarely reported. In this paper, the effects of flanking sequences on binding affinity between a series of unimolecular parallel-stranded G-quadruplex sequences derived from $c$-myc oncogene promoter (termed as $c$-myc G-quadruplexes) and their ligands are discussed in detail. The results showed that the flanking sequences on $c$-myc G-quadruplexes play key roles in (G-quadruplex)-ligand interaction. When a $c$-myc G-quadruplex is bound to its ligands, the flanking sequences might form a binding cavity above the terminal G-quartet, which could provide a suitable site for ligands to dock in. Moreover, the bases on flanking sequences could interact with ligand through $\pi-\pi$ stacking, and finally form a sandwich-stacking mode (terminal G-quartet, ligand and bases on the flanking sequence). This mode could stabilize the (G-quadruplex)-ligand complex effectively and enhance the binding affinity dramatically. However, flanking sequences are also found to exhibit steric hindrance effect which could impede the (G-quadruplex)-ligand binding.
\end{abstract}

G-quadruplex, flanking sequences, interaction mechanism, $c$-myc, cyanine dye

Citation: $\quad$ Gai W, Yang Q F, Xiang J F, et al. Roles of flanking sequences in the binding between unimolecular parallel-stranded G-quadruplexes and ligands. Chin Sci Bull, 2013, 58: 731-740, doi: 10.1007/s11434-012-5639-4

When DNA actively participates in biological process, including replication, transcription and recombination, it can adopt not only the regular Watson-Crick duplex, but some other special conformations, such as G-quadruplex [1-3], i-motif [4], Triad [5]. Immense studies in the past two decades have proved that G-rich tracts capable of forming Gquadruplex motif are found to be widespread in the promoter regions of several important genes that are involved in various cancers [6], as well as in telomeric repeat sequences which are involved in cancer cell proliferation [7,8]. Gquadruplex structure has been characterized in vitro [9], which is stabilized by Hoogsteen hydrogen bonding among

*Corresponding authors (email: tangyl@iccas.ac.cn; yangqf@iccas.ac.cn) four guanine bases arranged in a square planar configuration. Owing to the unique secondary motif, G-quadruplex is impervious to be attacked by enzymes targeting normal single-stranded or duplex DNA, and consequently is able to regulate the transcription and replication of specific gene. Therefore, G-quadruplex motif attracts growing interests and the ligands able to stabilize or regulate the formation of specific G-quadruplex motifs have been considered as potential novel anticancer medicines [10-12].

On the purpose of improving the stability of G-quadruplex, several interaction mechanisms between specific Gquadruplexes and their ligands have been investigated. There are two main binding modes have been reported so far, end-stacking mode [13-18] and groove binding mode 
[19-24]. In both binding modes, besides the core scaffold (G-quartets), the roles of loops are also important to interaction and have been addressed in many studies [25,26]. However, it is believed that G-quadruplexes motif cannot exist independently in vivo, but are embedded in the chromosome and are linked by flanking sequence. The elucidation of flanking sequences impact on the interaction between G-quadruplexes and their ligands could be helpful in aspects of understanding the binding mechanism and designing more selective and combinative G-quadruplex stabilizing agents. Unfortunately, few studies about the effect of flanking sequences have been reported so far.

Human $c$-myc is a transcription factor that is central to regulation of cell growth, proliferation, differentiation and apoptosis [27-33]. The aberrant overexpression of the $c-m y c$ gene is considered to be associated with the progression of many cancers [34]. C-myc executes its multiple activities mostly through transcriptional regulation of the target genes. There is an important element in the $c-m y c$ promoter region, termed nuclease-hypersensitivity element $\mathrm{III}_{1}\left(\mathrm{NHE} \mathrm{III}_{1}\right)$, which controls about $90 \%$ of total $c$-myc transcription. It is reported that $\mathrm{NHE} \mathrm{III}_{1}$ is a 27 bp guanine-rich sequence, located 142 to 115 base pairs upstream of the P1 promoter, containing six consecutive guanine stretches [35,36]. As a

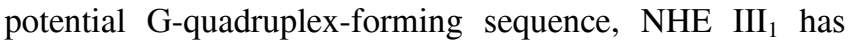
been proved to be able to form two different unimolecular $\mathrm{G}$-quadruplex conformations in equilibrium in $\mathrm{K}^{+}$solution $[37,38]$, the basket form (an antiparallel-stranded G-quadruplex possessing two lateral loops and a central diagonal loop) and the chair form (an parallel-stranded one with three propeller loops). The chair form has been shown to be kinetically and biologically favored as it is 3-fold more stable than the basket one in transcriptional activity of the $c-m y c$ promoter [38]. Then a $22 \mathrm{bp}$ variant of the $\mathrm{NHE} \mathrm{III}_{1}$, $5^{\prime}-\left[\mathrm{T}_{4} \mathrm{GA}-\left(\mathrm{G}_{7} \mathrm{G}_{8} \mathrm{G}_{9} \mathrm{TG}_{11} \mathrm{G}_{12} \mathrm{G}_{13} \mathrm{GAG}_{16} \mathrm{G}_{17} \mathrm{G}_{18} \mathrm{TG}_{20} \mathrm{G}_{21} \mathrm{G}_{22}\right)-\mathrm{G}\right.$ $\mathrm{AA}_{25}$ ]-3' (termed $c$-myc-2345), was designed which could form stable parallel-stranded chair form G-quadruplex structure in $\mathrm{K}^{+}$solution $[39,40]$. As shown in Figure 1, NMRbased folding topology of $c$-myc-2345 in $\mathrm{K}^{+}$solution had been resolved, which presents a typical unimolecular parallel-stranded G-quadruplex motif, including three G-tetrads, one two-residual loop $\left(\mathrm{G}_{14} \mathrm{~A}_{15}\right)$, two one-residual loops $\left(\mathrm{T}_{10}\right.$ and $\left.\mathrm{T}_{19}\right)$ and two three-residual flanking sequences $\left(\mathrm{T}_{4} \mathrm{G}_{5} \mathrm{~A}_{6}\right.$ at 5'-end and $\mathrm{G}_{23} \mathrm{~A}_{24} \mathrm{~A}_{25}$ at 3 '-end). Thanks to the stable Gquadruplex motif and the typical loop folding, c-myc-2345 is always considered as a model sequence in those in vitro (G-quadruplex)-ligands interaction studies [40].

Herein, this paper tries to investigate the impact of flanking sequences in detail in (G-quadruplex)-ligand binding by taking advantage of a series of designed sequences based on $c$-myc-2345, called $c$-myc G-quadruplexes. Being a large class of fluorescent pigment, cyanine dye has been used extensively in photosensitization, nonlinear optics, biological fluorescent detection, biomedicine and photodynamic therapy of tumor [41-45]. It is reported that cyanine

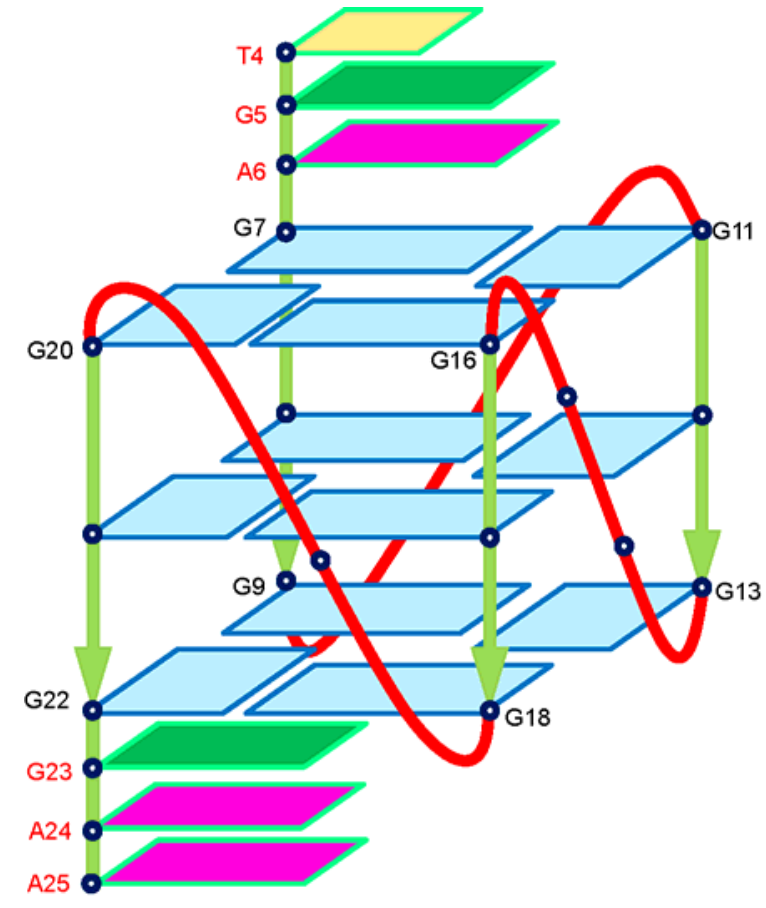

Figure 1 The NMR-based folding topology of $c-m y c-2345$.

dye MTC, 3,3'-di(3-sulfo-propyl)-4,5,4',5'-dibenzo-9-methylthiacarbocyanine triethylammonium salt ( as shown in Figure 2(a)), is an excellent G-quadruplex ligand and could present significant spectral changes and strong fluorescent enhancement when binding to specific G-quadruplex [46]. Besides, methylene blue (termed MB), 3,7-bis(dimethylamino)-phenothiazin-5-ium chloride (as shown in Figure 2(b)), is another kind of pigment whose fluorescence would be quenched when binding to specific G-quadruplex [47]. Since both MTC and MB binding to parallel G-quadruplex in end-stacking mode, this paper discussed the role of the flanking sequences of $c$-myc G-quadruplexes in the interaction with MTC/MB.

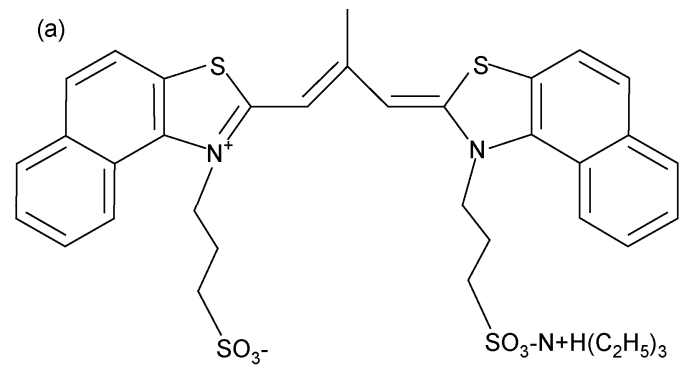<smiles>CN(C)C1=CC2SC3=CC(=[N+](C)C)C=CC3=NC2C=C1</smiles>

Figure 2 Structural formula of MTC (a) and MB (b). 


\section{Materials and methods}

\subsection{Materials and sample preparation}

The cyanine dye MTC was synthesized according to Hamer [48] and Fichen's [49] methods, and the purity was proved by mass spectrometry and nuclear magnetic resonance (NMR) (shown in Supporting Information). MB was purchased from Sigma (USA). All oligonucleotides were purchased from Sangon Biotech Co., Ltd. (Shanghai, China) and purified by PAGE (purity $98 \%$ ). Analytical grade methanol, $\mathrm{KH}_{2} \mathrm{PO}_{4}$, $\mathrm{K}_{2} \mathrm{HPO}_{4}$ and EDTA were purchased from Beijing Chem. Co. (China). Ultrapure water prepared by Milli-Q Gradient ultrapure water system (Millipore) was used throughout the experiments.

The stock solutions of MTC and MB were prepared by dissolving MTC and MB in methanol to $200 \mathrm{mmol} / \mathrm{L}$, respectively, and then storing in dark at $-4^{\circ} \mathrm{C}$ to prevent photodegradation. All designed $c-m y c$ G-quadruplexes were listed in Table 1. The stock solutions of all G-quadruplexes were prepared by dissolving them directly into PBS (20 mmol L ${ }^{-1} \mathrm{KH}_{2} \mathrm{PO}_{4} / \mathrm{K}_{2} \mathrm{HPO}_{4}, 1$ mmol L ${ }^{-1}$ EDTA, $\mathrm{pH} 7.4$ ), filtered through a microfiltration membrane, heated to $90^{\circ} \mathrm{C}$ for $5 \mathrm{~min}$ and then gradually cooled to room temperature at a rate of $0.3^{\circ} \mathrm{C} \mathrm{min}{ }^{-1}$. Concentrations of the stock solutions were determined by their absorbance at $260 \mathrm{~nm}$. All DNA samples had been stored for more than $24 \mathrm{~h}$ at $4^{\circ} \mathrm{C}$ and then structurally identified by circular dichroism (CD) spectra.

The measured sample was prepared by mixing a quantity of MTC solution with $c$-myc G-quadruplex solution, staying at $4^{\circ} \mathrm{C}$ for $1 \mathrm{~h}$, and then being diluted by PBS. Then the samples were kept in darkness overnight at $4^{\circ} \mathrm{C}$ before measurement in order to realize the full complexation.

\section{$1.2 \mathrm{UV} / \mathrm{Visible}-\mathrm{melting}$ experiments}

The measured samples were prepared by diluting DNA stock solution by PBS $\left(2 \mathrm{mmol} \mathrm{L}{ }^{-1} \mathrm{KH}_{2} \mathrm{PO}_{4} / \mathrm{K}_{2} \mathrm{HPO}_{4}, 1 \mathrm{mmol} \mathrm{L}{ }^{-1}\right.$ EDTA, pH 7.4). The ultraviolet absorption spectra were measured by an Agilent-8453 UV/visible spectrophotometer equipped with a Peltier effect heated cuvette holder in $10 \mathrm{~mm}$ quartz cells. A temperature range of $30-95^{\circ} \mathrm{C}$ was used to monitor the absorbance at $295 \mathrm{~nm}$ at a heating rate of $0.5^{\circ} \mathrm{C} \mathrm{min} \mathrm{mi}^{-1}$ with a $3 \mathrm{~min}$ holding time before each measurement.

\subsection{Fluorescence and circular dichroism (CD) spectra measurements}

Fluorescence and CD spectra were taken on a Hitachi F4500 spectrophotometer and a JASCO J-815 spectrophotometer, respectively, in $10 \mathrm{~mm}$ quartz cells at room temperature. Xenon arc lamp was used in the excitation light source in fluorescence measurement. The excitation wavelength was $530 \mathrm{~nm}$. Both excitation and emission slits were $5 \mathrm{~nm}$ and the scan speed was $240 \mathrm{~nm} \mathrm{~min}^{-1}$. All CD spectra
Table 1 Designed $c$-myc G-quadruplex oligonucleotides

\begin{tabular}{|c|c|c|c|}
\hline \multirow[b]{2}{*}{ Oligo name } & \multicolumn{3}{|c|}{ Sequence (from $5^{\prime}$ to $3^{\prime}$ ) } \\
\hline & $\begin{array}{c}\text { 5'-flanking } \\
\text { residues }\end{array}$ & Core G-rich sequence & $\begin{array}{c}\text { 3'-flanking } \\
\text { residues }\end{array}$ \\
\hline c-myc-core & - & GGGTGGGGAGGGTGGG & - \\
\hline$c$-myc-part & - & GGGTGGGGAGGGTGGG & GAA \\
\hline$c-m y c-2345$ & TGA & GGGTGGGGAGGGTGGG & GAA \\
\hline$c-m y c-1 A$ & A & GGGTGGGGAGGGTGGG & A \\
\hline$c-m y c-2 A$ & AA & GGGTGGGGAGGGTGGG & AA \\
\hline$c-m y c-3 A$ & AAA & GGGTGGGGAGGGTGGG & AAA \\
\hline$c-m y c-4 A$ & AAAA & GGGTGGGGAGGGTGGG & AAAA \\
\hline$c-m y c-5 A$ & AAAAA & GGGTGGGGAGGGTGGG & AAAAA \\
\hline$c-m y c-6 A$ & AAAAAA & GGGTGGGGAGGGTGGG & AAAAAA \\
\hline$c-m y c-1 T$ & $\mathrm{~T}$ & GGGTGGGGAGGGTGGG & $\mathrm{T}$ \\
\hline$c-m y c-A T$ & TA & GGGTGGGGAGGGTGGG & AT \\
\hline$c-m y c-A A T$ & TAA & GGGTGGGGAGGGTGGG & AAT \\
\hline$c-m y c-A A A T$ & TAAA & GGGTGGGGAGGGTGGG & AAAT \\
\hline
\end{tabular}

were collected at $1000 \mathrm{~nm} \mathrm{~min}{ }^{-1}$ between 190 and $400 \mathrm{~nm}$ at room temperature, with 5 scans averaged.

\subsection{Molecular modeling}

All the molecular modeling works and simulations were performed by using the Insight II 2005 software (Accelrys Inc., San Diego, CA, USA) on a DELL 5300 work station under CHARMM forcefield. The structure of MTC was first built by using the sketching module and then minimized for 500 steps by the steepest descent algorithm followed by 2000 steps of conjugated gradient algorithm. The topology structures of $c-m y c-1 A, c-m y c-2 A, c-m y c-3 A, c-m y c-4 A$, $c-m y c-5 A$ and $c-m y c-6 A$ were established respectively based on the crystal structure of $\mathrm{NHE} \mathrm{III}_{1}$ element obtained from the RCSB Protein Data Bank whose PDB ID was 1XAV. The binding sites of MTC to the $c$-myc G-quadruplexes have been revealed in our previous study by using NMR titration experiments [52]. So MTC was arranged directly to the binding site of the receptor and 5000 steps of minimization procedure of the receptor-ligand complex were performed by using the steepest descent algorithm. Then a 100 ps molecular dynamic simulation procedure was performed for each complex. In the dynamic simulation, the NVT assembly was used with Generalized Born model as the implicit solvent model. The simulation temperature was controlled around $300 \mathrm{~K}$ and the time step was $1 \mathrm{fs}$. The conformation of the complex was saved every $100 \mathrm{fs}$. During both the minimization and dynamic simulation process, the DNA bases which compose the flanking sequences were flexible while other bases were fixed. 


\section{Results}

\subsection{Verification of the secondary structures of all c-myc G-quadruplex sequences}

In aspects of exploring the effect of flanking sequences, it is essential to be sure that all G-quadruplex sequences form similar parallel-stranded G-quadruplex topologies like $c$-myc2345 shown in Figure 1. Therefore, a set of well-established methods were used in order to demonstrate the exact motifs of DNA samples. UV-melting experiments show that all sequences exhibit an invert transition at $295 \mathrm{~nm}$ (as shown in Supporting Information), which is a typical characterization of G-quadruplex denaturation [50], inferring that all DNA samples were folded into specific G-quadruplex motifs. Furthermore, the CD spectra of all sequences present a positive CD signal around $265 \mathrm{~nm}$ and a negative one at 240 $\mathrm{nm}$ (as shown in Supporting Information), which are typical parallel-stranded G-quadruplex features [51]. It is confirmed that all DNA samples with same core sequence folded into similar expected parallel-stranded G-quadruplex motifs.

\subsection{The binding characterization and stoichiometry of cyanine dye MTC to $c$-myc-core}

In our previous study, we have reported the binding characteristics of cyanine dye ETC which has similar molecular structure to MTC, to $c-m y c-2345$ [52]. It was found that ETC binds to $c$-myc-2345 by stacking on both ends of the parallel G-quadruplex and the propeller loops could facilitate the binding. In this study, we will firstly focus on the sequence $c$-myc-core (as shown in Table 1), which is part of

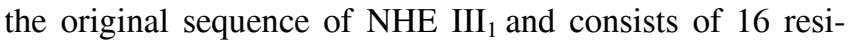
dues that are indispensable to form a integrate G-quadruplex structure. So, it is necessary to study the spectral features between $c$-myc-core and MTC though both $c$-myc-core and $c$-myc-2345 have the same G-quadruplex core sequence.

Due to the extended planar $\pi$-electron conjugated system, MTC tends to self-assemble in PBS and exhibits only a predominant absorption band at $660 \mathrm{~nm}$ assigned to J-aggregates [54-56]. Figure 3(a) shows the absorption spectra of $10 \mu \mathrm{mol} \mathrm{L}{ }^{-1}$ MTC with different concentrations of $c$-myccore. Presence of $c$-myc-core resulted in a gradual decrease in the absorbance of MTC J-aggregates at $660 \mathrm{~nm}$, accompanying with the appearance of a new peak located at $583.5 \mathrm{~nm}$, which could be assigned to MTC monomer. It is indicated that c-myc-core could interact with MTC and is able to disaggregate MTC J-aggregates into monomer.

Both MTC monomer and J-aggregate show weak fluorescence peaks at 600 and $664 \mathrm{~nm}$, respectively, as shown in Figure 3(b), the fluorescence intensity of MTC monomer could be strongly enhanced in the presence of $c$-myc-core and the fluorescence intensity increased simultaneously with increasing the ratio of $[c-m y c$-core $]:[\mathrm{MTC}]$. This behavior of cyanine dye MTC could be interpreted by the competi-
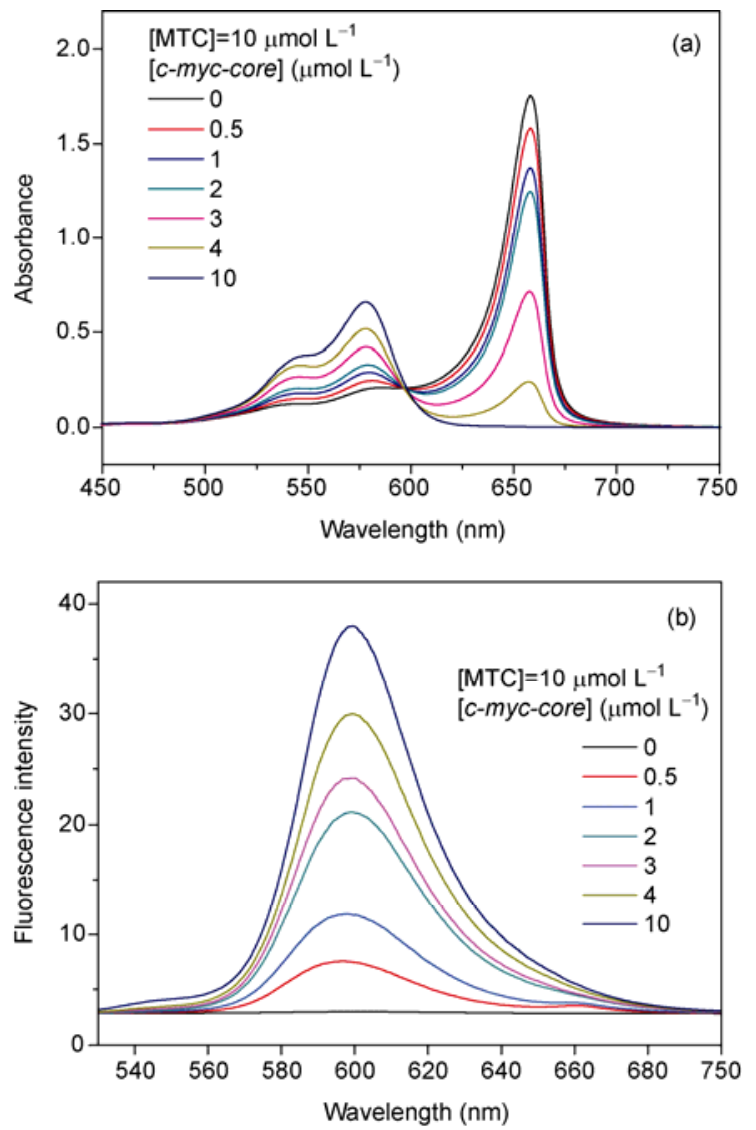

Figure 3 The absorption spectra (a) and fluorescence emission spectra (b) of $10 \mu \mathrm{mol} / \mathrm{L} \mathrm{MTC}$ in the presence of various concentrations of $c$-myc-core

tion between radiation transition and radiationless transition caused by facile rotation around methine bridge [57]. When MTC monomer is bound to $c$-myc-core, the rotation is hindered and radiationless transition is inhibited, thus resulting in the enhancement of MTC monomer's fluorescence intensity.

From the spectral characteristics between $c$-myc-core and MTC, we could see that $c$-myc-core is exactly the same as $c$-myc-2345 in aspects of interaction with cyanine dyes.

The binding ratio between $c$-myc-core and MTC was also calculated by fluorescence titration experiment using the method introduced by Bean et al. [53]. As shown in Figure 4 , the job curve (dash lines) was demonstrated to intercross at about 0.9 , indicating that the binding ratio of MTC and $c$-myc-core is close to $1: 1$. However, in our previous research, the binding ratio of ETC and $c$-myc-2345 is 2:1 [52]. The result is interesting since $c$-myc-core and c-myc-2345 have the same core sequence and can fold into similar parallel G-quadruplex motifs which could provide similar binding sites (two terminal G-quartets) for MTC/ETC to stack on. The only difference between them is that $c-m y c-2345$ has two 3-nt sequences flanked on its terminal G-quartets while c-myc-core does not. The results indicate that the flanking sequences of $c-m y c-2345$ might also play important roles in the (c-myc-2345)-MTC interaction. 


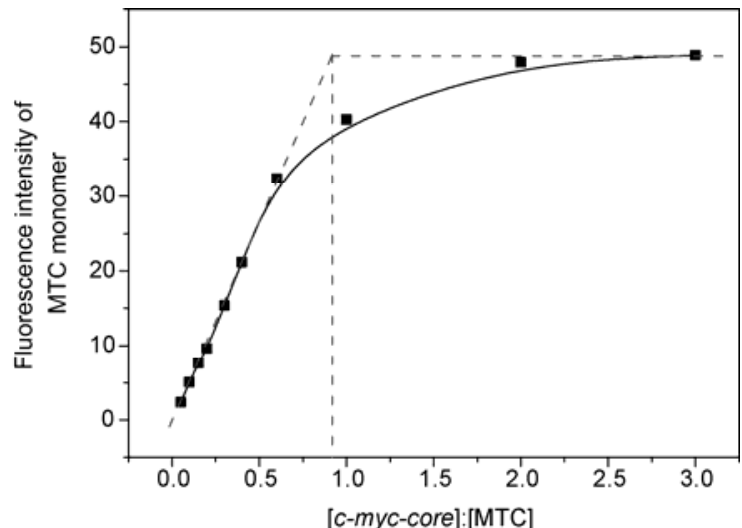

Figure 4 The change of $10 \mu \mathrm{mol} \mathrm{L}{ }^{-1}$ MTC monomer fluorescence intensity at $600 \mathrm{~nm}$ against the ratio of [c-myc-core $]$ :[MTC]. Stoichiometry was obtained by tangents method [53].

\subsection{Effect of the flanking sequences in the interaction between ( $c$-myc G-quadruplexes) and MTC/MB}

In order to explore the effect of flanking sequences in the interaction of $c$-myc G-quadruplex and its ligands in detail, a series of sequences derived from $c$-myc-core were designed (as shown in Table 1). Compared with $c$-myc-core, $c$-mycpart has a 3 '-end flanking sequence GAA while $c$-myc-2345 has two flanking sequences on both ends (TGA at $5^{\prime}$-end and GAA at 3 '-end), whose structure has been elucidated clearly by Phan and Ambrus et al. [40,58,59].

As shown in Figure 5(a), compared with c-myc-core, $c$ myc-part induced sharper changes while $c$-myc-2345 could induce the sharpest changes of MTC monomer fluorescence intensity under the same [c-myc G-quadruplexes]/[MTC] ratio, indicating the order of binding affinity to MTC is c-myc-core (without flanking sequence) $<c$-myc-part (with 5 '-end flanking sequence) $<c-m y c-2345$ (with both 5 '-end and 3 '-end flanking sequences). Furthermore, the Job curves (dash lines) of c-myc-core, c-myc-part and c-myc-2345 intercrossed at $0.90,0.78$ and 0.55 , respectively, indicating that their binding ratio to MTC are close to $1: 1,1.5: 1$ and $2: 1$, respectively.

In order to confirm whether the flanking sequences play common role in (c-myc G-quadruplexes)-ligand binding, the interaction between the $c$-myc G-quadruplexes and another ligand MB was also studied. Compared to MTC, MB has a smaller planar aromatic ring and it can also end-stack on specific G-quadruplex. Besides, it is reported that the fluorescence intensity of $\mathrm{MB}$ would be quenched in the presence of specific G-quadruplex [47]. To compare the binding affinities of $c$-myc G-quadruplexes to their ligands, the fluorescence intensities of MTC and MB on the ratio of [c-myc G-quadruplexes]:[ligands] $=0.6$ had been discussed. As shown in Figure 5(b), the fluorescence intensity of MTC monomer increases in the order of c-myc-core, $c$-myc-part and c-myc2345 while the fluorescence intensity of MB decreases in the same order (the detailed fluorescence titration spectra
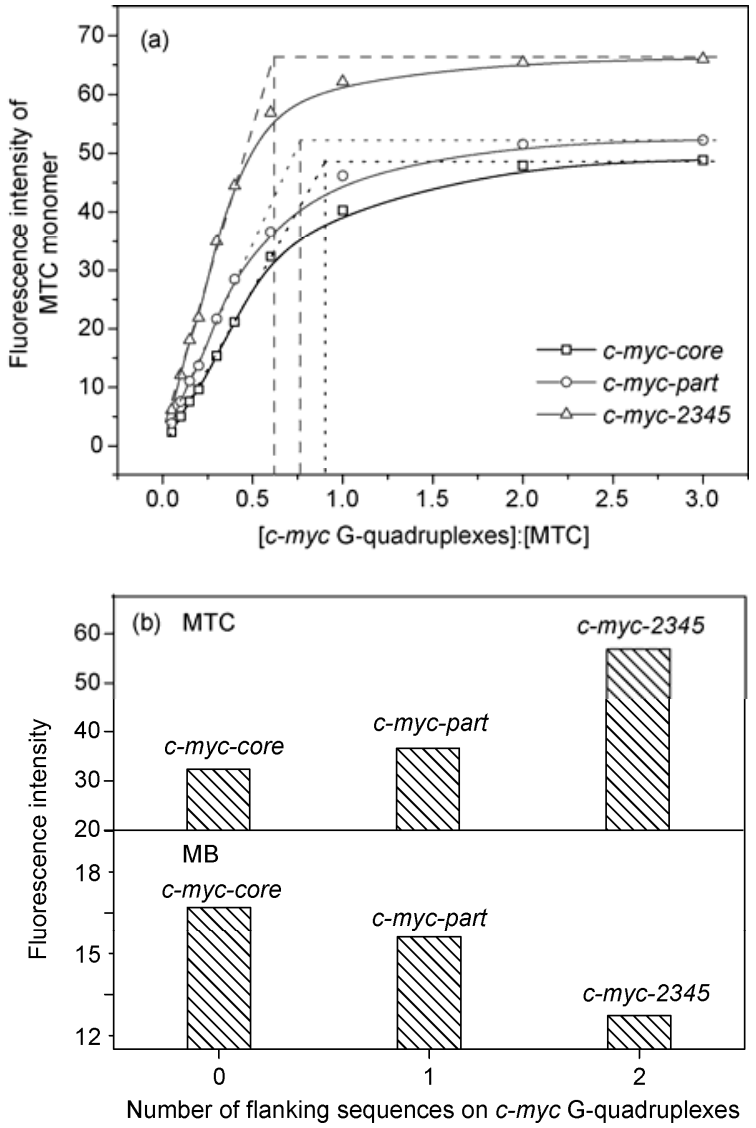

Figure 5 (a) The change of $10 \mu \mathrm{mol} \mathrm{L}{ }^{-1}$ MTC monomer fluorescence intensity at $600 \mathrm{~nm}$ against the ratio of [c-myc G-quadruplexes]:[MTC]. Stoichiometries were obtained by tangents method [53]. (b) Fluorescence intensity of MTC monomer at $600 \mathrm{~nm}$ (up) and MB at $678 \mathrm{~nm}$ (down) in the presence of various $c$-myc G-quadruplexes on the ratio of [c-myc G-quadruplexes]:[MTC/MB] $=0.6$, respectively.

for $\mathrm{MB}$ were shown in supporting information), indicating that the $c$-myc G-quadruplexes with different number of flanking sequences presents similar order of binding affinity to MTC/MB, c-myc-core $<c-m y c-$ part $<c-m y c-2345$.

However, as revealed by Kettani et al. [60-62], some G-quadruplexes without flanking sequences, such as $c$-myccore, are likely to form end-to-end stacking dimeric-quadruplex scaffold by two monomers. In that case, the decrease of $c$-myc-core/MTC binding ratio might be partly caused by dimerization effect because of the competitiveness between quadruplex-quadruplex dimerization and quadruplex-MTC interaction. Considering that, we compare the following two sequences: $c$-myc-core and $c-m y c-1 T$. Apparently, $c$-myc-1T has an extensive thymine base attached on the G-quadruplex core, which would then inhibit the intermolecular dimerization effect. Thus, if the decrease of $c$-myc-core/MTC binding ratio is caused completely by dimerization, $c-m y c-1 T$ is supposed to exhibit a higher binding ratio to MTC than c-myc-core for its weak dimerization effect. As shown in Figure 6, the fluorescence titration experiment demonstrates 


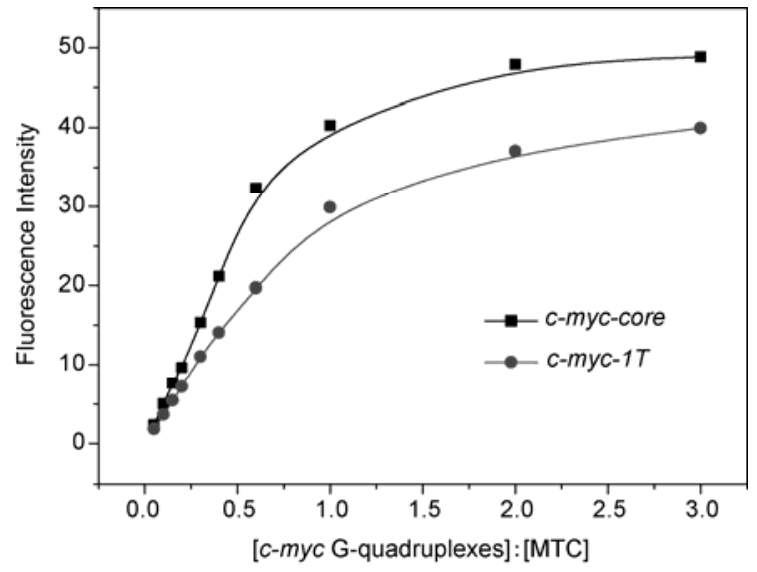

Figure 6 The change of $10 \mu \mathrm{mol} \mathrm{L}{ }^{-1}$ MTC monomer fluorescence intensity at $600 \mathrm{~nm}$ against the ratio of [c-myc G-quadruplexes]: [MTC].

that the sequence $c$-myc-IT exhibits even lower binding affinity than $c$-myc-core, which is opposite to the former expected result. Thus, we prove that the flanking sequence do play important roles in the interaction between $c$-myc G-quadruplexes and MTC.

\section{Discussion}

It is supposed to discuss about the fact that MTC binds to $c$-myc-core with a ratio of $1: 1$ though both ends of it are almost equally exposed for MTC. From the view of binding dynamics, there is an equilibrium between the "boundMTC" and "unbound-MTC" upon MTC-quadruplex interaction. Though there are two sites for MTC to stack on, it doesn't mean that the "observed stoichiometry" is supposed to be equal to the number of binding sites because of the exist of equilibrium. And it is not the case that each binding site inevitably accommodates one ligand molecule because of the exist of dynamic equilibrium. The higher the binding affinity is, the closer to 1.0 the observed binding stoichiometry is for each binding site. Thus, the observed binding ratio could be variable between quadruplex and MTC and is determined by not only the number of binding site but also the equilibrium shift.

On the basis of previous work and the above results, it is considered that there are two primary driving forces in the interaction between MTC and these $c$-myc G-quadruplexes, the $\pi-\pi$ stacking between the terminal G-quartet and the aromatic rings of MTC, and the facilitating effect of flanking sequence. Patel et al. [59] and Armbrus et al. [58,63] had proposed that flanking sequences could form a stable fold-back stacking conformation and cap the terminal Gtetrads very well, which appears to play a key role in stabilizing G-quadruplex structures. Tang and co-workers [52] also had proved that specific terminal loop might fold into a suitable binding cavity, just like flanking sequences in $c-m y c$ G-quadruplexes do, could facilitate the (G-quadruplex)- ligand interaction. That implies two factors would be involved in the facilitating effect of flanking sequences, steric match and noncovalent interaction. On one hand, flanking sequences are highly likely to fold into specific steric cavity above the terminal G-quartet to accommodate the ligand. On the other hand, there is potentially $\pi-\pi$ stacking interaction between aromatic planar of ligand and those of nucleotide residues in flanking sequences, which could provide further noncovalent interaction for the $(c-m y c$ G-quadruplexes)-ligand complex. In order to improve our hypothesis, these two factors have been discussed in detail.

\subsection{Flanking sequences facilitate binding by forming a suitable binding cavity}

If a suitable binding cavity forms, it is easy to understand that the facilitating effect would increase with the extension of flanking sequences since short flanking sequence is not long enough to fold into a proper cavity. Therefore, a series of sequences with certain flanking sequence extension based on $c$-myc-core, $c$-myc- $1 A-c-m y c-6 A$ (as shown in Table 1) were designed to determine the formation of suitable binding cavity in the (c-myc G-quadruplex)-ligand interaction.

It should be noticed the fact that the binding affinity is proportional to fluorescence intensity is only reasonable in case of the same binding ratio, or more accurately, the same binding site or binding mode. Obviously it is inaccurate to compare the binding affinity of a ligand to two macromolecules simply by using fluorescence intensity if there are more than one different binding sites on the two macromolecules for a ligand. However, in this specific case when MTC interacts with the unimolecular parallel G-quadruplexes, the MTC could only stack on the 5'- or 3'-end G-quartets and could not adopt intercalation or groovebinding mode, which has been proved in our former work. Since there are the same number of binding sites for those G-quadruplexes in this study, the observed binding ratios in our experiments is completely dominated by the boundunbound ligand equilibrium. Therefore, the extent of tightness of MTC upon binding to G-quadruplexes, namely the binding affinity, could be reasonably reflected and represented by the fluorescence intensity of MTC here free of considering the binding stoichiometry.

Figure 7 shows the MTC monomer and MB fluorescence intensities in the presence of various $c$-myc G-quadruplexes on the ratio of $[c-m y c$ G-quadruplexes] : [MTC/MB] $=0.6$. For MTC, higher fluorescence intensity means stronger binding affinity to $c$-myc G-quadruplexes, while for $\mathrm{MB}$ lower fluorescence intensity means stronger binding affinity to $c$-myc G-quadruplexes. It is observed that in the case of both MTC and MB, $c-m y c-1 A$ exhibits weaker binding affinity than $c$-myc-core, which indicates flanking chain with just one adenine residue hinders the interaction of $c-m y c$ G-quadruplex and its ligand. When the flanking chain extends to two to five residues, an enhancement in binding 

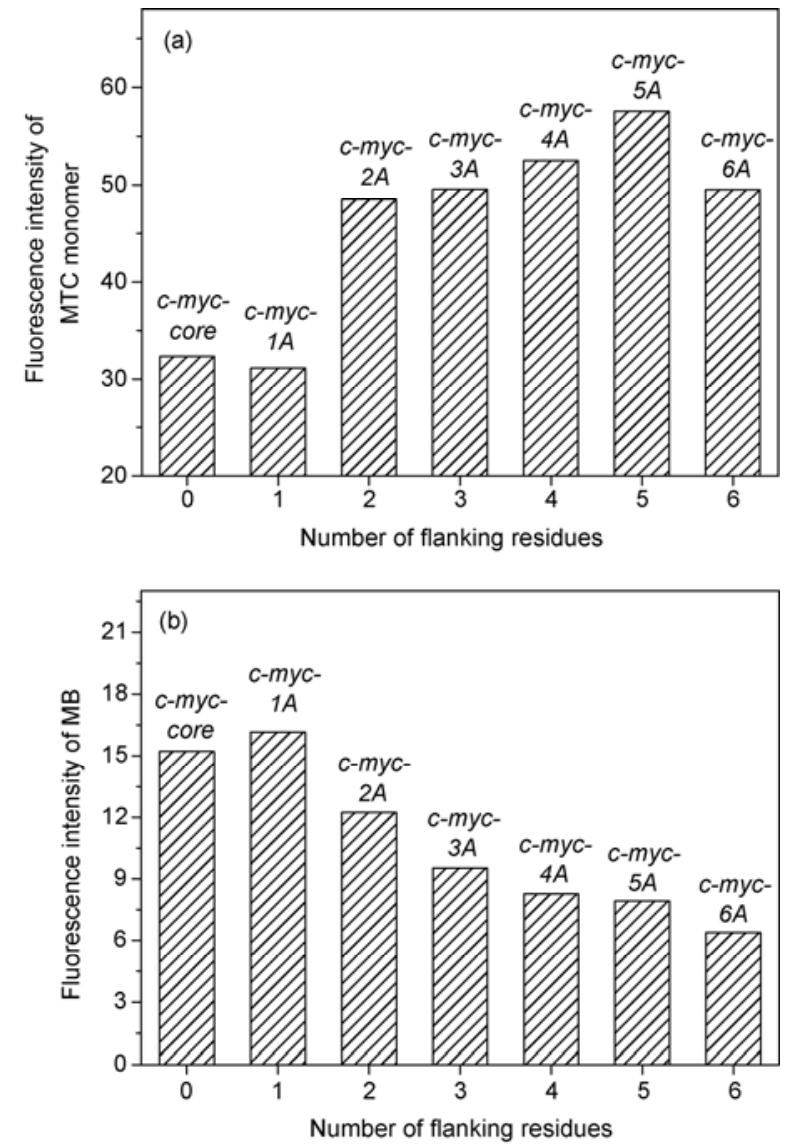

Figure 7 The fluorescence intensity of $10 \mu \mathrm{mol} \mathrm{L}^{-1}$ MTC monomer (a) and $\mathrm{MB}$ (b) in the presence of various $c$-myc G-quadruplexes on the ratio of $[c-m y c$ G-quadruplexes] : [MTC/MB] $=0.6$, respectively.

affinities could be found in the case of both MTC and MB, and the affinities increased regularly with the extension of flanking sequence, implying that extension of flanking sequences is in favor of the formation of a suitable binding cavity. For $c-m y c-6 A$, it exhibits weaker binding ability to MTC than $c$-myc-5A, inferring overlong flanking sequence might induces higher steric hindrance and block MTC accessing to the terminal G-quartet, and consequently decrease the binding ability of $c-m y c-6 A$ to MTC. However, in the case of $\mathrm{MB}$, owing to the smaller molecular frame than MTC, it could easily access to the terminal G-quartet of $c-m y c-6 A$ and exhibits higher binding ability to $c-m y c-6 A$ than that to $c-m y c-5 A$.

The results reveal that flanking sequences provide not only the positive facilitating effect but also the negative hindrance effect to the ( $c$-myc G-quadruplexes)-ligands binding. In order to further confirm the formation of binding cavity and visualize the hindrance effect of flanking sequences, molecular modeling method was used to provide a deep view for the (c-myc G-quadruplexes)-MTC complexes.

As shown in Figure 8, it is observed that the flanking residue in $c$-myc- $1 A$ leans against the terminal G-quartet and hardly stack with MTC. So the flanking chain with only one
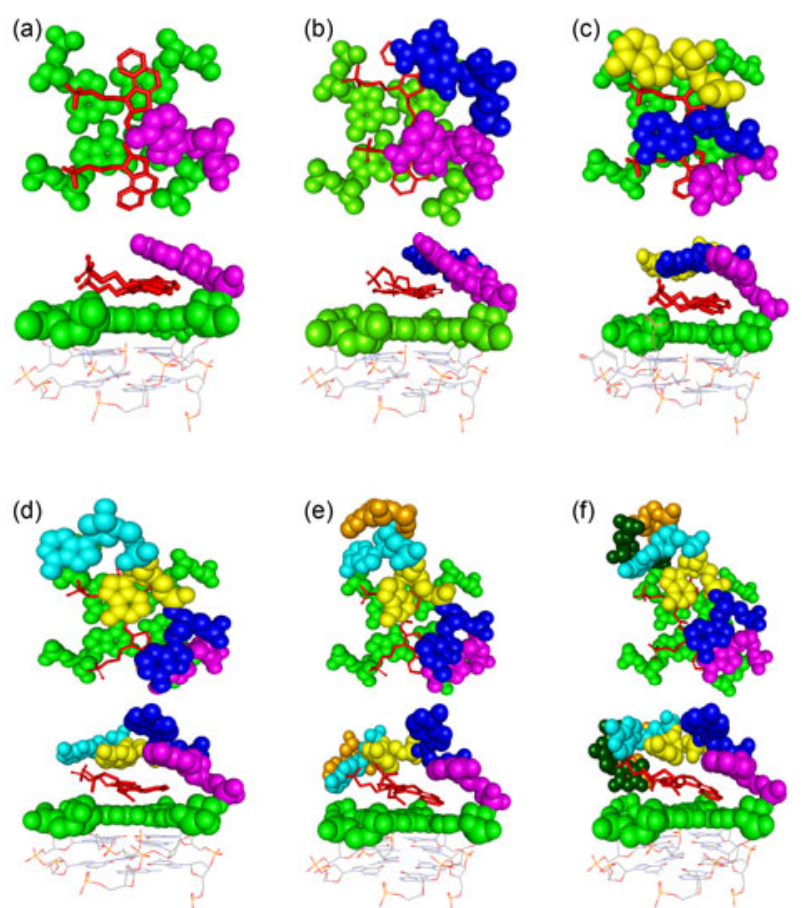

Figure 8 The the structure of (c-myc G-quadruplexes)-MTC complexes obtained by Insight II 2005 software (upper: top view; lower: lateral view), (a) (c-myc-1A)-MTC, (b) (c-myc-2A)-MTC, (c) (c-myc-3A)-MTC, (d) (c-myc-4A)-MTC, (e) (c-myc-5A)-MTC, (f) (c-myc-6A)-MTC. The color of terminal G-tetrad is light green, the MTC is red and the first to the sixth flanking residue is magenta, blue, yellow, cyan, golden and dark green, respectively.

adenine residue primarily shows steric hindrance effect. When the second adenine residue was added, its purine ring is able to stack with the aromatic ring of MTC and to dramatically stabilize the $(c-m y c-2 A)$-MTC complex. With the extension of the flanking sequence, it tends to fold into a cavity topology structure together with the G-quartet that could accommodate MTC and enhance the binding affinity. Thus, from $c-m y c-2 A$ to $c-m y c-5 A$, additional stacking ability and suitable binding cavity provided by the flanking sequences can help "snatching" MTC molecule and lead to gradual increase in ( $c$-myc G-quadruplexes)-MTC binding affinities. To $c-m y c-6 A$, six flanking adenine residues above the G-tetrad results in higher steric hindrance so that the binding affinity weakens.

In a word, the flanking sequence is potential to form a binding cavity to accommodate the ligand so as to facilitate the (c-myc G-quadruplex)-MTC binding. And the length of the flanking sequence is important. Too long or too short of the flanking sequence would lead to unsuitable binding site and high steric hindrance, and consequently decrease the binding affinity.

\subsection{Flanking sequences facilitate binding by a sandwich- stacking system}

As discussed above, the flanking sequence was prone to 
form a cavity which could help (c-myc G-quadruplexes)ligand binding and the aromatic ring may provide extra $\pi-\pi$ stacking force in the interaction. Therefore, a sandwichstacking mode is probably formed in the (c-myc G-quadruplexes)-ligand complexes, which consists of three planar units, the base planar ring (purine or pyrimidine ring) of the flanking residue, aromatic planar ring of MTC/MB and the terminal G-quartet.

To prove this, four variant sequences, $c-m y c-1 T, c-m y c-$ $A T, c-m y c-A A T$ and c-myc-AAAT (as shown in Table 1), whose flanking sequences contain a mutated terminal thymine residue respectively comparing with $c$-myc- $1 A$ to $c-m y c-4 A$, were designed. Owing to larger electron conjugated planar and richer electron density, it is reasonable that purine could provide stronger $\pi-\pi$ stacking interaction to MTC than pyrimidine, which means adenine residue in the flanking sequence may help "snatching" MTC molecule closer than thymine. In this point of view, $c$-myc G-quadruplexes with flanking sequences consisting of pure adenines are supposed to exhibit higher binding affinity than those with terminal $\mathrm{A} \rightarrow \mathrm{T}$ mutation.

Figure 9 shows the fluorescence intensity of MTC monomer in the presence of various $c$-myc G-quadruplexes with two groups of the flanking sequences, the pure-A group and terminal $\mathrm{A} \rightarrow \mathrm{T}$ mutation group. Similar to the pure-A group, $c-m y c-1 T$ also shows lower binding affinity to MTC than $c$-myc-core since the flanking sequence with only one thymine residue is too short to fold into a suitable cavity for MTC to bind and primarily presents hindrance effect. With the extension of the flanking sequence, from $c-m y c-1 T$ to $c-m y c-A A A T$, their binding affinities to MTC gradually increase owing to the formation of a favorable binding cavity and sandwich-stacking system.

It is easy to observe that for the $c-m y c$ G-quadruplexes with the same flanking sequence length, the $\mathrm{A} \rightarrow \mathrm{T}$ mutation

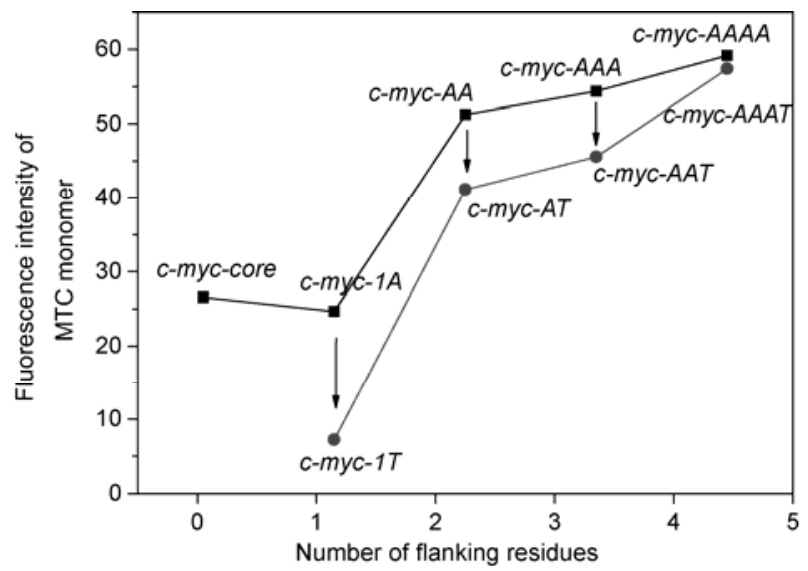

Figure 9 Fluorescence intensity of $10 \mu \mathrm{mol} \mathrm{L}{ }^{-1}$ MTC monomer in the presence of various $c$-myc G-quadruplexes with different flanking sequences on the ratio of $[c-m y c$ G-quadruplexes $]:[\mathrm{MTC}]=0.6$, whose terminal residue is adenine ( $\mathbf{\square})$ or thymine (e). group shows lower binding affinities to MTC than the pureA one, indicating that compared with adenine, the terminal thymine residue is less favorable for the (c-myc G-quadruplexes)-MTC binding. The result is consistent with our hypothesis on the formation of sandwich-stacking mode in the interaction. Furthermore, it is noticed that in the case of the c-myc G-quadruplexes with four residues flanking sequence, $c-m y c-A A A T$ presents almost the same binding affinity to MTC as $c-m y c-4 A$, inferring that the second and third residues of flanking sequence might be the key sites which are involved in the sandwich-stacking mode.

In summary, when a $c$-myc G-quadruplex is bound to its ligands, the flanking sequence can form a binding cavity by forming a fold-back structure above the terminal G-quartet which could provide a suitable site for ligands to dock in and form a sandwich-stacking mode (terminal G-quartet, ligand and bases on the flanking sequence). This mode could stabilize the (c-myc G-quadruplex)-ligand complex effectively and increase the binding affinity dramatically.

\section{Conclusion}

In this study, the critical roles of flanking sequences in the interaction between $c$-myc G-quadruplexes and their ligands have been proved and details of how the flanking sequences take part in the interaction were discussed according to the experiment results.

It is found that $c$-myc G-quadruplex with two flanking tails exhibits the strongest binding affinity to its ligands, while that with only one tail does stronger than that without any flanking tail in the case of both MTC and MB. On one hand, it is partially caused by quadruplex dimerization. On the other hand, it is because the flanking sequences have the potential to enhance the c-myc-part and c-myc-2345-ligand binding.

Furthermore, it is demonstrated that when a $c$-myc Gquadruplex is bound to its ligands, the flanking sequence is prone to form a binding cavity by forming a fold-back structure above the terminal G-quartet which could provide a suitable site for ligands to dock in and tends to form a sandwich-stacking mode together with ligand and terminal G-quartet. This mode could stabilize the (c-myc G-quadruplex)-ligand complex effectively and increase the binding affinity dramatically. On the other hand, flanking sequences also exhibit certain steric hindrance effect to impede the (c-myc G-quadruplexes)-ligands binding.

1 Hurley L H, Siddiqui-Jain A, Grand C L, et al. Direct evidence for a G-quadruplex in a promoter region and its targeting with a small molecule to repress $c$-myc transcription. Proc Natl Acad Sci USA, 2002, 99: 11593-11598

2 Sun D Y, Thompson B, Cathers B E, et al. Inhibition of human telomerase by a G-quadruplex-interactive compound. J Med Chem, 1997, 40: 2113-2116

3 Neidle S, Burge S, Parkinson G N, et al. Quadruplex DNA: Sequence, 
topology and structure. Nucleic Acids Res, 2006, 34: 5402-5415

4 Xiao J H, Davidson I, Macchi M, et al. In vitro binding of several cell-specific and ubiquitous nuclear proteins to the Gt-I motif of the sv40 enhancer. Gene Dev, 1987, 1: 794-807

5 Kuryavyi V V, Jovin T M. Triad-DNA-A model for trinucleotide repeats. Nat Genet, 1995, 9: 339-341

6 Huppert J L, Balasubramanian S. G-quadruplexes in promoters throughout the human genome. Nucleic Acid Res, 2007, 35: 406413

7 Wang Y, Patel D J. Guanine residues in d(T2AG3) and d(T2G4) form parallel-stranded potassium cation stabilized G-quadruplexes with antiglycosidic torsion angles in solution. Biochemistry, 1992, 31: $8112-8119$

8 Izbicka E, Nishioka D, Marcell V, et al. Telomere-interactive agents affect proliferation rates and induce chromosomal destabilization in sea urchin embryos. Anti Canc Drug Des, 1999, 14: 355-365

9 Williamson J R. G-quartet structures in telomeric DNA. Annu Rev Biophys Biomol Struct, 1994, 23: 703-730

10 Parkinson G N, Ghosh R, Neidle S. Structural basis for binding of porphyrin to human telomeres. Biochemistry, 2007, 46: 2390-2397

11 Han F X G, Wheelhouse R T, Hurley L H. Interactions of TMPyP4 and TMPyP2 with quadruplex DNA. Structural basis for the differential effects on telomerase inhibition. J Am Chem Soc, 1999, 121: 35613570

12 Seenisamy J, Bashyam S, Gokhale V, et al. Design and synthesis of an expanded porphyrin that has selectivity for the $c$-myc Gquadruplex structure. J Am Chem Soc, 2005, 127: 2944-2959

13 Dash J, Waller Z A E, Pantos G D, et al. Synthesis and binding studies of novel diethynyl-pyridine amides with genomic promoter DNA G-quadruplexes. Chem-Eur J, 2011, 17: 4571-4581

14 Mcguire R, Mcmillin D R. Steric effects direct the binding of porphyrins to tetramolecular quadruplex DNA. Chem Commun, 2009, 7393-7395

$15 \mathrm{Xu} \mathrm{W}$, Tan J H, Chen S B, et al. Studies on the binding of 5-Nmethylated quindoline derivative to human telomeric G-quadruplex. Biochem Biophys Res Commun, 2011, 406: 454-458

16 Pilch D S, Barbieri C M, Rzuczek S G, et al. Targeting human telomeric G-quadruplex DNA with oxazole-containing macrocyclic compounds. Biochimie, 2008, 90: 1233-1249

17 Rahman K M, Reszka A P, Gunaratnam M, et al. Biaryl polyamides as a new class of DNA quadruplex-binding ligands. Chem Commun, 2009, 4097-4099

18 Sparapani S, Bellini S, Gunaratnam M, et al. Bis-guanylhydrazone diimidazo 1,2-a:1,2-c pyrimidine as a novel and specific G-quadruplex binding motif. Chem Commun, 2010, 46: 5680-5682

19 Dash J, Shirude P S, Hsu S T D, et al. Diarylethynyl amides that recognize the parallel conformation of genomic promoter DNA Gquadruplexes. J Am Chem Soc, 2008, 130: 15950-15956

20 Martino L, Virno A, Pagano B, et al. Structural and thermodynamic studies of the interaction of distamycin A with the parallel quadruplex structure $\left[\mathrm{d}(\mathrm{TGGGGT)}]_{4}\right.$. J Am Chem Soc, 2007, 129: 16048-16056

21 Sun H X, Tang Y L, Xiang J F, et al. Spectroscopic studies of the interaction between quercetin and G-quadruplex DNA. Bioorg Med Chem Lett, 2006, 16: 3586-3589

22 Baraldi P G, Bovero A, Fruttarolo F, et al. DNA minor groove binders as potential antitumor and antimicrobial agents. Med Res Rev, 2004, 24: 475-528

23 Nelson S M, Ferguson L R, Denny W A. Non-covalent ligand/DNA interactions: Minor groove binding agents. Mutat Res, 2007, 623. 24-40

24 Li Q, Xiang J F, Li X D, et al. Stabilizing parallel G-quadruplex DNA by a new class of ligands: Two non-planar alkaloids through interaction in lateral grooves. Biochimie, 2009, 91: 811-819

25 Guedin A, Gros J, Alberti P, et al. How long is too long? Effects of loop size on G-quadruplex stability. Nucleic Acids Res, 2010, 38: 7858-7868

26 Arora A, Maiti S. Stability and molecular recognition of quadruplexes with different loop length in the absence and presence of molecular crowding agents. J Phys Chem B, 2009, 113: 8784-8792

27 Kelly K, Cochran B H, Stiles C D, et al. Cell-specific regulation of the $c$-myc-gene by lymphocyte mitogens and platelet-derived growthfactor. Cell, 1983, 35: 603-610

28 Evan G I, Wyllie A H, Gilbert C S, et al. Induction of apoptosis in fibroblasts by c-myc protein. Cell, 1992, 69: 119-128

29 Kinzler K W, He T C, Sparks A B, et al. Identification of $c-m y c$ as a target of the APC pathway. Science, 1998, 281: 1509-1512

30 Steller H. Mechanisms and genes of cellular suicide. Science, 1995, 267: 1445-1449

31 Cosconati S, Marinelli L, Trotta R, et al. Tandem application of virtual screening and NMR experiments in the discovery of brand new DNA quadruplex groove binders. J Am Chem Soc, 2009, 131: 16336

32 Pelengaris S, Khan M. The c-myc oncoprotein as a treatment target in cancer and other disorders of cell growth. Expert Opin Ther Target, 2003, 7: 623-642

33 Gartel A L, Shchors K. Mechanisms of c-myc-mediated transcriptional repression of growth arrest genes. Exp Cell Res, 2003, 283: 17-21

34 Slamon D J, Dekernion J B, Verma I M, et al. Expression of cellular oncogenes in human malignancies. Science, 1984, 224: 256-262

35 Siebenlist U, Hennighausen L, Battey J, et al. Chromatin structure and protein binding in the putative regulatory region of the $c$-myc gene in Burkitt lymphoma. Cell, 1984, 37: 381-391

36 Davis T L, Firulli A B, Kinniburgh A J. Ribonucleoprotein and protein factors bind to an H-DNA-forming $c$-myc DNA element: Possible regulators of the $c-m y c$ gene. Proc Natl Acad Sci USA, 1989, 86: 9682-9686

37 Simonsson T, Pecinka P, Kubista M. DNA tetraplex formation in the control region of c-myc. Nucleic Acids Res, 1998, 26: 1167-1172

38 Siddiqui-Jain A, Grand C L, Bearss D J, et al. Direct evidence for a G-quadruplex in a promoter region and its targeting with a small molecule to repress c-myc transcription. Proc Natl Acad Sci USA, 2002, 99: 11593-11598

39 Patel D J, Phan A T, Kuryavyi V. Human telomere, oncogenic promoter and 5'-UTR G-quadruplexes: Diverse higher order DNA and RNA targets for cancer therapeutics. Nucleic Acids Res, 2007, 35 : 7429-7455

40 Phan A T, Modi Y S, Patel D J. Propeller-type parallel-stranded G-quadruplexes in the human c-myc promoter. J Am Chem Soc, 2004, 126: 8710-8716

41 James T H, Mees C E K. The Theory of the Photographic Process. 4th ed. New York: Macmillan, 1977

42 Salvioli S, Ardizzoni A, Franceschi C, et al. JC-1, but not DiOC6(3) or rhodamine 123 , is a reliable fluorescent probe to assess delta psi changes in intact cells: Implications for studies on mitochondrial functionality during apoptosis. FEBS Lett, 1997, 411: 77-82

43 Blatchford J W, Gustafson T L, Epstein A J, et al. Spatially and temporally resolved emission from aggregates in conjugated polymers. Phys Rev B Conden Matt, 1996, 54: R3683-R3686

44 Li N, Yu C, Huang F. Novel cyanine-AMP conjugates for efficient 5' RNA fluorescent labeling by one-step transcription and replacement of [gamma-32P]ATP in RNA structural investigation. Nucleic Acid Res, 2005, 33: e37

45 Davila J, Harriman A, Gulliya K S. Photochemistry of merocyanine 540: The mechanism of chemotherapeutic activity with cyanine dyes. Photochem Photobiol, 1991, 53: 1-11

46 Yang Q, Xiang J F, Yang S, et al. Verification of intramolecular hybrid/parallel G-quadruplex structure under physiological conditions using novel cyanine dye $\mathrm{H}$-aggregates: Both in solution and on $\mathrm{Au}$ film. Anal Chem, 2010, 82: 9135-9137

47 Sun H X, Xiang J F, Zhang Y Z, et al. Spectroscopic studies of the interaction between methylene blue and G-quadruplex. Chin Sci Bull, 2006, 51: 1687-1692

48 Hamer F M. The Cyanine Dyes and Related Compounds. New York: Interscience Publishers, 1964

49 Ficken G E. The Chemistry of Synthetic Dyes. New York: Academic Press, 1971

50 Mergny J L, Phan A T, Lacroix L. Following G-quartet formation by 
UV-spectroscopy. FEBS Lett, 1998, 435: 74-78

51 Balagurumoorthy P, Brahmachari S K, Mohanty D, et al. Hairpin and parallel quartet structures for telomeric sequences. Nucleic Acid Res, 1992, 20: 4061-4067

52 Yang Q F, Xiang J F, Yang S, et al. Verification of specific G-quadruplex structure by using a novel cyanine dye supramolecular assembly: II. The binding characterization with specific intramolecular G-quadruplex and the recognizing mechanism. Nucleic Acid Res, 2010, 38: 1022-1033

53 Bean R C, Shepherd W C, Kay R E, et al. Spectral changes in a cationic dye due to interaction with macromolecules. III. Stoichiometry and mechanism of the complexing reaction1. J Phy Chem, 1965, 69: 4368-4379

54 Mcrae E G, Kasha M. Enhancement of phosphoescence ability upon aggregation of dye molecules. J Chem Phys, 1958, 28: 721-722

55 Herz A H. Aggregation of sensitizing dyes in solution and their adsorption onto silver halides. Adv Colloid Interface Sci, 1977, 8: 237-298

56 Chen C P, Zhou B M, Li D H, et al. Electron-transfer events in solutions of cyanine dyes. J Photochem Photobiol A Chem, 1995, 89: 25-29

57 Guo C, Xiang J, Feng J, et al. Effect of $\mathrm{TiO}_{2}$ colloids on the fluorescence behavior of two cyanine dyes. J Colloid Interface Sci,
2002, 246: 401-409

58 Ambrus A, Chen D, Dai J X, et al. Solution structure of the biologically relevant g-quadruplex element in the human $c$-myc promoter implications for G-quadruplex stabilization. Biochemistry, 2005, 44: 20482058

59 Phan A T, Kuryavyi V, Gaw H Y, et al. Small-molecule interaction with a five-guanine-tract G-quadruplex structure from the human MYC promoter. Nat Chem Biol, 2005, 1: 167-173

60 Kettani A, Basu G, Gorin A, et al. A two-stranded template-based approach to G.(C-A) triad formation: Designing novel structural elements into an existing DNA framework. J Mol Biol, 2000, 301: 129-146

61 Kettani A, Bouaziz S, Skripkin E, et al. Interlocked mismatch-aligned arrowhead DNA motifs. Structure, 1999, 7: 803-815

62 Kettani A, Gorin A, Majumdar A, et al. A dimeric DNA interface stabilized by stacked A.(G.G.G.G).A hexads and coordinated monovalent cations. J Mol Biol, 2000, 297: 627-644

63 Zhang Z J, Dai J X, Veliath E, et al. Structure of a two-G-tetrad intramolecular G-quadruplex formed by a variant human telomeric sequence in $\mathrm{K}^{+}$solution: Insights into the interconversion of human telomeric G-quadruplex structures. Nucleic Acids Res, 2010, 38: 1009-1021

Open Access This article is distributed under the terms of the Creative Commons Attribution License which permits any use, distribution, and reproduction in any medium, provided the original author(s) and source are credited.

\section{Supporting Information}

Figure S1 The MS-ESI spectrum of cyanine dye MTC

Table S1 Elemental analysis of MTC

Figure S2 The numbering scheme of molecular structure of MTC

Figure S3 The ${ }^{1} \mathrm{H}-\mathrm{NMR}$ spectrum of cyanine dye MTC in DMSO-d

Table S2 The full assignments of the proton peaks of MTC

Figure S4 The absorption spectra of MTC monomer in methanol. Insert gives the curve of the absorbance at $573 \mathrm{~nm}$ against the concentration of MTC monomer.

Figure S5 CD spectra for all the $13 c-m y c$ G-quadruplexes.

Figure S6 UV-melting profiles for all the $13 c$-myc G-quadruplexes.

Figure S7 The fluorescence intensity of MTC monomer as a function of [c-myc G-quadruplexes]: [MTC]. (a) MTC vs. $c-m y c-1 A-c-m y c-6 A$; (b) MTC vs. $c-m y c-1 T, c-m y c-A T, c-m y c-A A T$ and $c-m y c-A A A T$.

Figure S8 The fluorescence intensity of MB as a function of [c-myc G-quadruplexes] : [MB]. (a) MB vs. $c$-myc-core, $c$-myc-part and $c$-myc-2345; (b) $\mathrm{MB}$ vs. $c-m y c-1 A-c-m y c-6 A$.

The supporting information is available online at csb.scichina.com and www.springerlink.com. The supporting materials are published as submitted, without typesetting or editing. The responsibility for scientific accuracy and content remains entirely with the authors. 